[7] H. Samavati, H. R. Rategh, and T. H. Lee, "A 5-GHz CMOS wireless LAN receiver front end," IEEE J. Solid-State Circuits, vol. 35, pp. 765-772, May 2000.

[8] G. D. Vendelin, A. Pavio, and U. L. Rohde, Microwave Circuit Design Using Linear and Nonlinear Techniques. New York: Wiley, 1990, ch. 6.

[9] J. W. M. Rogers, J. A. Macedo, and C. Plett, "The effect of varactor nonlinearity on the phase noise of completely integrated VCO's," IEEE J. Solid-State Circuits, vol. 35, pp. 1360-1367, Sept. 2000.

[10] C. P. Yue and S. S. Wong, "On-chip spiral inductors with patterned ground shields for Si-based RF IC's," IEEE J. Solid-State Circuits, vol. 33, pp. 743-752, May 1998.

[11] B. Razavi, RF Microelectronics. Englewood Cliffs, NJ: Prentice-Hall, 1998.

[12] D. B. Leeson, "A simple model of feedback oscillator noise spectrum," Proc. IEEE, pp. 329-330, Feb. 1966.

[13] T. H. Lee, The Design of CMOS Radio Frequency Integrated Circuits. Cambridge, U.K.: Cambridge Univ. Press, 1998.

[14] J. Rogers, L. Tan, T. Smy, N. Tait, and N. G. Tarr, "A high $Q$ on-chip $\mathrm{Cu}$ inductor post process for $\mathrm{Si}$ integrated circuits," Int. Interconnect Technol. Conf., pp. 239-241, June 1999.

[15] A. M. Niknejad and R. G. Meyer, "Analysis, design, and optimization of spiral inductors and transformers for Si RF IC's," IEEE J. Solid-State Circuits, vol. 33, pp. 1470-1481, Oct. 1998.

[16] J. R. Long and M. A. Copeland, "The modeling, characterization, and design of monolithic inductors for silicon RF IC's," IEEE J. Solid-State Circuits, vol. 32, pp. 357-369, Mar. 1997.

[17] M. Danesh, J. R. Long, R. A. Hadaway, and D. L. Harame, "A $Q$-factor enhancement technique for MMIC inductors," in IEEE RFIC Symp. Dig., June 1998, pp. 217-200.

[18] J. Craninckx, M. Steyaert, and H. Miyakawa, "A fully integrated spiral-LC CMOS VCO with prescaler for GSM and DCS-1800 systems," Custom Integrated Circuit Conf., pp. 403-406, May 1997.

\section{The Summation-by-Parts Algorithm-A New Efficient Technique for the Rapid Calculation of Certain Series Arising in Shielded Planar Structures}

\author{
Juan R. Mosig and A. Alvarez Melcón
}

\begin{abstract}
This paper presents a new technique for the convergence acceleration of a large class of series often arising in electromagnetic problems. The technique is based on the recursive application of the integration-by-parts technique to discrete sequences, thus the given name of the "summation-by-parts" technique. It is shown that the new technique greatly enhances the convergence rate of the series treated, and very small relative errors are obtained by performing a few simple operations. The new technique is applied to the efficient numerical calculation of the Green's functions in a parallel-plate waveguide.
\end{abstract}

Index Terms-Convergence acceleration, modal expansions, shielded Green's functions.

\section{INTRODUCTION}

In many electromagnetic problems, the relevant physical quantities (electric and magnetic fields) and associated quantities (potentials) are expressed in terms of infinite series that are usually very slow conver-

Manuscript received April 5, 2000.

J. R. Mosig is with the Laboratory of Electromagnetics and Acoustics, Swiss Federal Institute of Technology, CH-1015 Lausanne, Switzerland (e-mail juan.mosig@epfl.ch).

A. Alvarez Melcón is with the Technical University of Carthagene, E-30202 Cartagena, Spain

Publisher Item Identifier S 0018-9480(02)00735-4. gent. This is the case, for instance, when analyzing shielded circuits [1], cavity-backed antennas [2], or microwave devices inside photonic crystals [3]. In these problems, the need of numerically evaluating infinite sums, usually very time-consuming, prevents, in general, the development of efficient software codes.

To try to overcome this difficulty, several convergence series acceleration techniques have been developed in the past. Detailed and complete survey on series acceleration is available in any modern textbook (see, e.g., [4]).

As for the application of these general methods to the acceleration of series arising in electromagnetic problems, a very interesting work can be found in [5]. In that paper, some results on series acceleration are given when these techniques are applied to the numerical evaluation of the free-space periodic Green's functions, and to the evaluation of the quasi-static Green's function term developed within the complex images representation derived in [6].

Other acceleration techniques employed in the past include the use of the Poisson's summation formula together with the Kummer's transformation for the evaluation of the shielded Green's functions [7], [8] and the Ewald transformation, which was used in [9] for the same purpose.

In spite of all these efforts, the use of these techniques in a reliable systematic fashion is not straightforward, and some situations and geometries can be found where the efficiency of the algorithms breaks down. In this context, this paper describes an alternative algorithm to accelerate the convergence behavior of certain series often arising in electromagnetic problems. It is shown in this paper that the new technique can be applied in a reliable fashion, and greatly accelerates the convergence rate of the series treated.

\section{THEORY}

In many microwave circuits involving multilayered planar shielded configurations, the relevant Green's functions of the problem can be formulated in terms of very slow convergent modal series of the form

$$
S_{\infty}=\sum_{n=0}^{\infty} \tilde{G}_{n} f_{n}
$$

where $\tilde{G}_{n}$ represents the spectral-domain Green's function of the problem, which it is a slow varying function, and $f_{n}$, which is a highly oscillatory (typically sinusoidal) function. In this section, we will present the mathematical transformations leading to the new formulation for the convergence acceleration of the series whose general form is shown in (1).

To start, we first define partial sums and reminders of the original series as follows:

$$
\begin{aligned}
S_{N-1} & =\sum_{n=0}^{N-1} \tilde{G}_{n} f_{n} \\
R_{N} & =\sum_{n=N}^{\infty} \tilde{G}_{n} f_{n} \\
S_{\infty} & =S_{N-1}+R_{N}
\end{aligned}
$$

and since the partial sums $S_{N-1}$ are bounded, the attention must be focused in the efficient evaluation of the infinite remainder $R_{N}$. To do so, we apply simple algebraic manipulations to the remainder $R_{N}$ in (2), allowing us to rewrite it as

$$
\begin{aligned}
& R_{N} \\
& =\sum_{n=N}^{\infty} \tilde{G}_{n} f_{n}=\tilde{G}_{N} \sum_{n=N}^{\infty} f_{n}+\sum_{n=N}^{\infty}\left[\left(\tilde{G}_{n+1}-\tilde{G}_{n}\right)\left(\sum_{k=n+1}^{\infty} f_{k}\right)\right] .
\end{aligned}
$$


The important change when transforming (2) into (3) is that now we have written the residue $R_{N}$ in a new form, where only differences of Green's functions $\tilde{G}_{n}$ and partial sums of sinusoidal terms $f_{n}$ do appear.

The key point here is that we expect that a straightforward analytical procedure can apply to obtain the partial sums of $f_{n}$ as closed formulas. This, and the fact that the remaining terms of the series should exhibit good convergence properties, being differences between successive values of a slowly varying function, show the numerical interest of the procedure.

It is now clear that the same procedure can be applied again to the sum in the right-hand side of (3) since it has the same formal structure than the original sum defining the residual $R_{N}$ in (2). We obtain

$$
R_{N}=\sum_{n=N}^{\infty} \tilde{G}_{n}^{(0)} f_{n}^{(0)}=\sum_{i=1}^{\infty} \tilde{G}_{N}^{(1-i)} f_{N-1}^{(+i)}
$$

where we have defined the successive sums of $f_{n}$ and differences of $\tilde{G}_{n}$ in the following form:

$$
\begin{aligned}
\tilde{G}_{n}^{(0)} & =\tilde{G}_{n} \\
\tilde{G}_{n}^{(-i)} & =\tilde{G}_{n+1}^{(1-i)}-\tilde{G}_{n}^{(1-i)} \\
f_{n}^{(0)} & =f_{n} \\
f_{n}^{(+i)} & =\sum_{k=n+1}^{\infty} f_{k}^{(i-1)}, \quad \text { for all } i=1,2,3,4, \ldots
\end{aligned}
$$

It is interesting to observe that the successive difference functions $\tilde{G}_{n}^{(-i)}$ can be viewed as the implementation of a numerical derivative, and the successive sum functions $f_{n}^{(+i)}$ as the implementation of a numerical integration. Having this is mind, it is not difficult to note that the expression given in (4) could also be directly obtained if a discrete equivalent of the integration-by-parts procedure is formally applied to the original expression of the residue $R_{N}$ in (2).

\section{MATHEMATICAL EXAMPLE}

In order to illustrate the numerical behavior of the new technique, we are now going to show how it can be applied to the summation of the following simple one-dimensional sinusoidal series [10]:

$$
S_{\infty}=\sum_{n=1}^{\infty} \frac{\sin (n x)}{n}=\frac{\pi-x}{2}, \quad 0<x<2 \pi .
$$

If one ignores the analytical solution of the series and tries a direct brute-force numerical evaluation of the sum, then a very slowly convergence behavior is found, and relative errors in the final sum greater than $1 \%$ are obtained, even after having summed more than 100 terms. Let us now try to apply the summation-by-parts technique given in (4), and see how the convergence of the series is affected. First note that by identifying (6) with (1), the following relations are established:

$$
\tilde{G}_{n}=\tilde{G}_{n}^{(0)}=\frac{1}{n} \quad f_{n}=f_{n}^{(0)}=\sin (n x) .
$$

For this selection of $f_{n}$, the series in (5b) can be easily evaluated analytically using simple trigonometric series formula, thus obtaining

$$
f_{n}^{(+i)}=\sum_{k=n+1}^{\infty} f_{k}^{(i-1)}=\frac{\cos \left\{(i-1) \frac{\pi}{2}+\left[n+i \frac{1}{2}\right] x\right\}}{2^{i}\left[\sin \left(\frac{x}{2}\right)\right]^{(i)}} .
$$

Using the above analytical expression, the relative errors for this series were computed when several correcting summation-by-parts terms are included in the expression of the remainder. Fig. 1 presents the results obtained, showing a dramatic improvement in the convergence for each

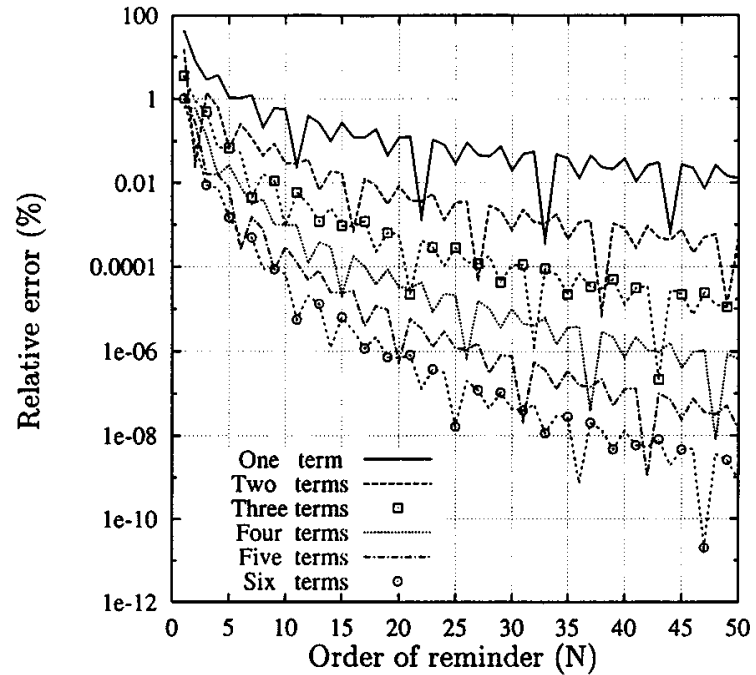

Fig. 1. Relative error for the series in (6) when different summation-by-parts correcting terms are used, and as a function of the order of the reminder $(N)$. For this calculation $x=2$.

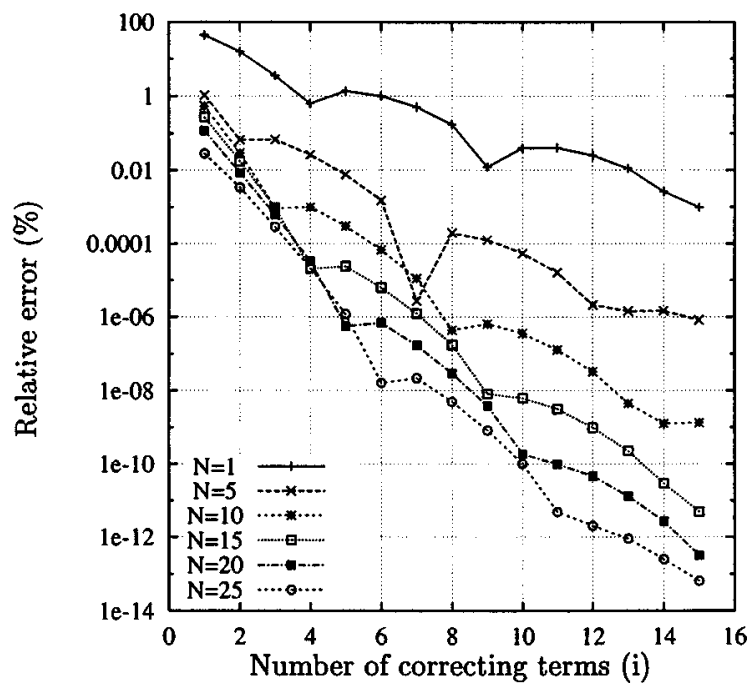

Fig. 2. Relative error for the series in (6) when different orders of the reminder are used, and as a function of the number of summation-by-parts terms $(i)$. For this calculation $x=2$.

new correcting term included. In particular, when six terms are used, an impressive numerical precision of $10^{-8} \%$ is obtained for values of the index $(N)$ of only $N=25$. In contrast, the convergence of the original series is very slow.

As a final convergence study of the novel technique developed, the relative error of the same series has been evaluated as a function of the number of correcting terms included $(i)$, when the order of the remainder $(N)$ is fixed constant. Fig. 2 presents the results obtained, indicating an impressive convergence rate, and that very accurate results are obtained with a very small order of recursive operations. However, it should be pointed out that the new algorithm is less efficient for very small values of $x$ since (8) then starts to show a divergence behavior, as indicated by the sine function in the denominator [11].

\section{Electromagnetic APPLICATION}

The technique derived can be used for the efficient numerical evaluation of slow convergent series arising in many electromagnetic problems. One such situation corresponds to the Green's functions inside a 


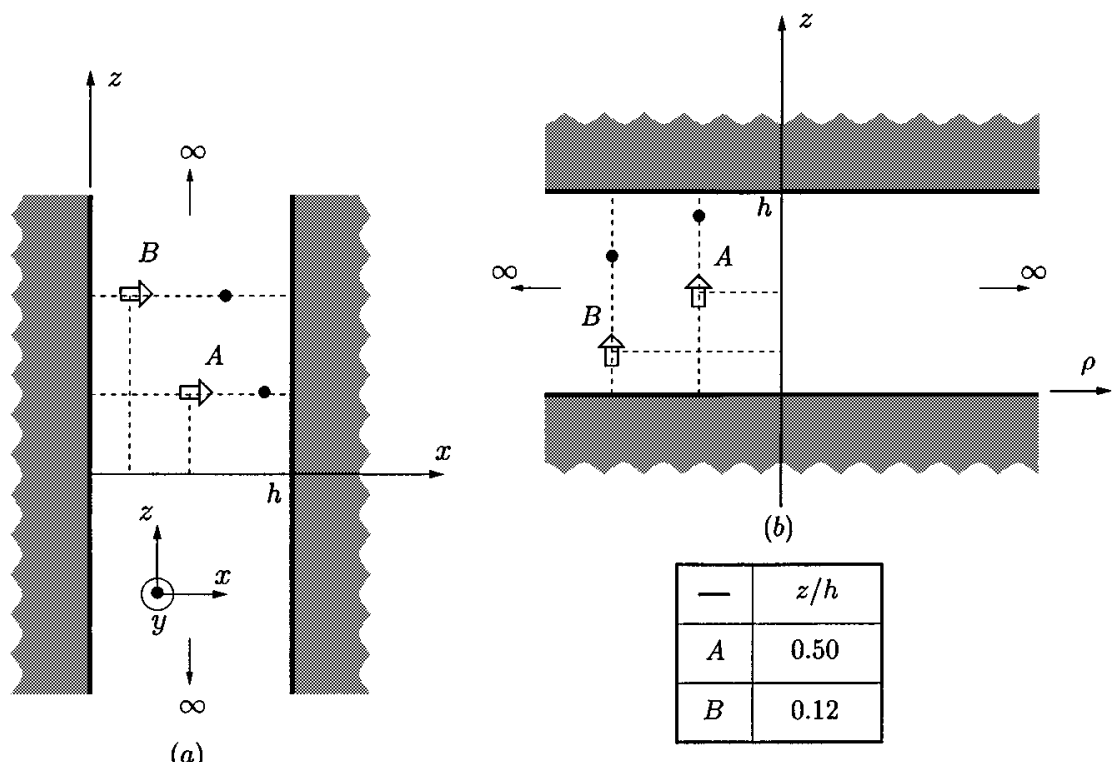

Fig. 3. Basic parallel-plate waveguide structure whose Green's functions are investigated. (a) Waveguide approach: fields are expressed as a sum of discrete waveguide modes propagating along $z$. (b) Stratified media approach: the plates are perpendicular to $z$ and fields are expanded into a continuous radial $k_{\rho}$ spectrum (Sommerfeld).

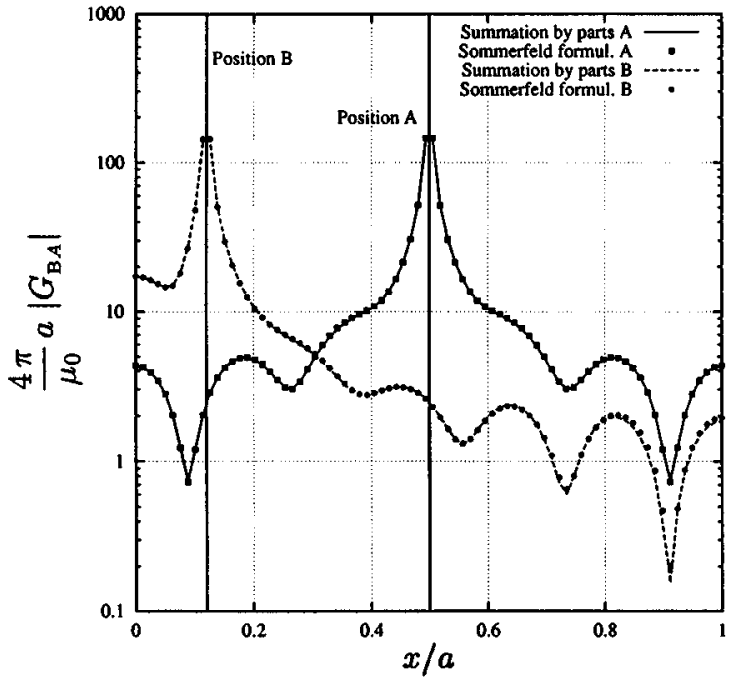

(a)

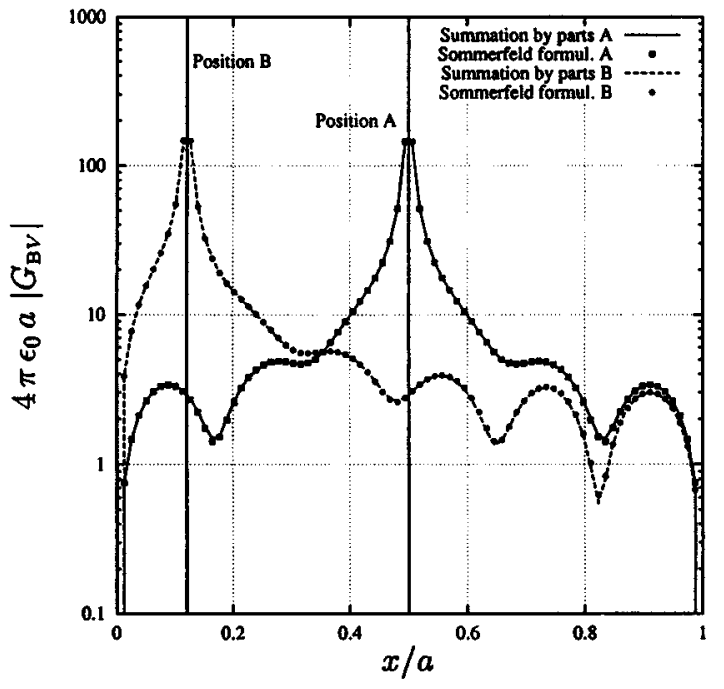

(b)

Fig. 4. Mixed-potential Green's functions for the parallel-plate structure shown in Fig. 3 when the source point is placed at positions A and B. Comparisons between the summation-by-parts technique and standard Sommerfeld formalism are included. Frequency: $45 \mathrm{GHz}(h=19.05 \mathrm{~mm})$. (a) Magnetic vector potential. (b) Electric scalar potential.

parallel-plate waveguide, as shown in Fig. 3. It is interesting to note that the analysis of this structure can be attempted in two different ways.

In the so-called waveguide approach, the propagation direction $z$ is parallel to the plates, and the field is expanded into a discrete spectrum of waveguides modes [see Fig. 3(a)]. This approach yields for the electric scalar potential [12]

$$
\begin{aligned}
4 \pi \epsilon_{0} G_{V}^{\mathrm{PPW}}(\rho) & =\frac{2 j \pi}{h} \sum_{n=1}^{\infty} H_{0}^{(2)}\left(\rho k_{z}\right) \sin \left(k_{x} x\right) \sin \left(k_{x} x^{\prime}\right) \\
k_{z} & =\sqrt{k_{0}^{2}-k_{x}^{2}} \\
\rho & =\sqrt{\left(z-z^{\prime}\right)^{2}+\left(y-y^{\prime}\right)^{2}} .
\end{aligned}
$$

The alternative approach (stratified media approach) defines the propagation direction $z$ as normal to the plates [see Fig. 3(b)]. We have now an unbounded medium terminated by two ground planes.
The field expansion gives a continuous spectrum of radial modes and is given by a Sommerfeld integral [13]

$$
\begin{aligned}
G_{V}^{\mathrm{PPW}}(\rho) & =\int_{0}^{\infty} J_{0}\left(k_{\rho} \rho\right) k_{\rho} \tilde{G}_{V}\left(k_{\rho}\right) d k_{\rho} \\
\rho & =\sqrt{\left(x-x^{\prime}\right)^{2}+\left(y-y^{\prime}\right)^{2}} .
\end{aligned}
$$

The numerical evaluation of this Green's function using any of the two formulations is, in general, not easy, due to the very slow convergence rates of the series and integrals involved. The problem of the numerical evaluation of the Sommerfeld integral in (10) has been extensively studied in the past, and very efficient algorithms are now available [13], [14]. Finally, with the technique derived in this paper, an efficient numerical evaluation of (9) can be attempted. In this case, the summation-by-parts technique just developed is used for the evaluation of the slow convergent infinite modal series. 
As an example, Fig. 4 shows the results obtained for the mixed-potential Green's functions in Fig. 3(a) obtained with (9), as compared with the Green's functions obtained using a standard Sommerfeld formulation (10) of the identical problem in Fig. 3(b). Fig. 4 shows the comparison for both the electric scalar potential and magnetic vector potential (analogous to the electric scalar potential, but not vanishing in the walls), when the source is placed at points A and B in Fig. 3, and as a function of the position of the observer point. As we observe, the agreement is excellent; therefore, validating the parallel-plate Green's functions computed with the new algorithm.

\section{CONCLUSIONS}

In this paper, a new and efficient technique for the convergence acceleration of a large class of series arising in electromagnetic problems has been presented. The technique can be viewed as the successive application of the integration-by-parts technique to discrete sequences; therefore, the given name of the "summation-by-parts technique."

The technique has been first applied to the numerical evaluation of a simple canonical series, showing that convergence is greatly enhanced, allowing to obtain very small relative errors with just a few operations. The technique described can be applied to many real electromagnetic problems and, in this paper, it has been used for the efficient numerical calculation of the parallel-plate mixed-potential Green's functions.

\section{REFERENCES}

[1] C. J. Railton and S. A. Meade, "Fast rigorous analysis of shielded planar filters," IEEE Trans. Microwave Theory Tech., vol. 40, pp. 978-985, May 1992.

[2] J. Y. Lee, T. S. Horng, and N. G. Alexopoulos, "Analysis of cavitybacked aperture antennas with a dielectric overlay," IEEE Trans. Antennas Propagat., vol. 42, pp. 1556-1562, Nov. 1994.

[3] A. A. Maradudin and A. R. McGurn, "Photonic band structures of twodimensional dielectric media," in Photonic Band Gaps and Localization. ser. NATO ASI series, C. M. Soukoulis, Ed. New York: Plenum, 1993, pp. 247-268.

[4] C. Brezinski and M. R. Zaglia, Extrapolation Methods. Amsterdam, The Netherlands: Elsevier, 1991.

[5] N. Kinayman and M. I. Aksun, "Comparative study of acceleration techniques for integrals and series in electromagnetic problems," Radio Sci., vol. 30, pp. 1713-1722, Nov.-Dec. 1995.

[6] Y. L. Chow, J. J. Yang, D. G. Fang, and G. E. Howard, "A closed-form spatial Green's function for the thick microstrip substrate," IEEE Trans. Microwave Theory Tech., vol. 39, pp. 588-592, Mar. 1991.

[7] G. V. Eleftheriades, J. R. Mosig, and M. Guglielmi, "A fast integral equation technique for shielded planar circuits defined on nonuniform meshes," IEEE Trans. Microwave Theory Tech., vol. 44, pp. 2293-2296, Dec. 1996.

[8] G. G. Gentili, L. E. Garcia-Castillo, M. Salazar-Palma, and F. PerezMartinez, "Green's function analysis of single and stacked rectangular microstrip patch antennas enclosed in a cavity," IEEE Trans. Antennas Propagat., vol. 45, pp. 573-579, Apr. 1997.

[9] M. J. Park and S. Nam, "Rapid calculation of the Green's function in the shielded planar structures," IEEE Microwave Guided Wave Lett., vol. 7, pp. 326-328, Oct. 1997.

[10] I. S. Gradshteyn and I. M. Ryzhik, Table of Integrals, Series, and Products. New York: Academic, 1965

[11] A. Alvarez-Melcón and J. R. Mosig, "Two techniques for the efficient numerical calculation of the Green's functions for planar shielded circuits and antennas," IEEE Trans. Microwave Theory Tech., vol. 48, pp. 1492-1504, Sept. 2000.

[12] J. J. Yang, Y. L. Chow, G. E. Howard, and D. G. Fang, "Complex images of an electric dipole in homogeneous and layered dielectrics between two ground planes," IEEE Trans. Microwave Theory Tech., vol. 40, pp. 595-600, Mar. 1992

[13] J. R. Mosig, "Integral equation technique," in Numerical Techniques for Microwave and Millimeter-Wave Passive Structures, T. Itoh, Ed. New York: Wiley, 1989, ch. 3, pp. 133-213.
[14] M.-J. Tsai, C. Chen, and N. G. Alexopoulos, "Sommerfeld integrals in modeling interconnects and microstrip elements in multi-layered media," Electromagnetics (Special Issue), vol. 18, no. 3, pp. 267-288, 1998

\section{Direct Extraction of Linear HBT-Model Parameters Using Nine Analytical Expression Blocks}

Achour Ouslimani, Jean Gaubert, Habiba Hafdallah, Ahmed Birafane, Pierre Pouvil, and H. Leier

\begin{abstract}
A method to determine the heterojunction bipoloar transistor (HBT) equivalent-circuit elements without numerical optimizations is presented. It is based on the extraction of nine analytical expressions, which are referred to here as "blocks." The model elements are extracted using certain blocks for some of them and three nonlinear equations derived from a combination of four expression blocks for some others. The base and collector resistances can be determined at each bias point. The method is validated treating the on-wafer HBTs.
\end{abstract}

Index Terms-Analytical method, heterojunction bipolar transistor, parameter extraction.

\section{INTRODUCTION}

An accurate parameter-extraction procedure of the linear equivalent circuit is crucial to optimize the device and circuit performances. Approaches that consist of reducing the number of unknown elements by using complementary characterizations for independently estimating as many heterojunction bipolar transistor (HBT) parameters as possible have been proposed [1]-[3]. Measurements of special test structures and geometrical and material parameters are needed in [1] to obtain some of the parasitic parameter values. The measurements of two separate test structures are proposed in [2] and [3] to determine the probe-pattern parasitic. The cutoff mode measurements are used in [2] to determine some of the HBTs' capacitances, and the equivalent circuit of the device biased to active mode is obtained using numerical optimizations. Analytical expressions and numerical optimizations are used in [3] and [4] and the information about the geometry device is used to evaluate the ratio $\mu$ between the extrinsic and intrinsic base-collector capacitances. Analytical extraction methods have been proposed in [5] and [6]. In [5], the method is based on the use of local fitting routines and certain assumptions. In [6], the $S$-parameters measured under open-collector condition are used to determine the extrinsic elements. An approach based on both empirical optimization and analytical evaluations is reported in [7]. Initial values of HBT parameters are estimated from dc and multibias $S$-parameter measurements and then used to achieve the evaluation of the HBT model elements by the impedance-block conditioned optimization. Finally, a procedure combining the analytical and optimization approaches was developed in [8], in which the influence of pad capacitances is discussed.

Manuscript received May 1, 2000; revised October 20, 2000.

A. Ouslimani, J. Gaubert, H. Hafdallah, A. Birafane, and P. Pouvil are with the Ecole Nationale Supérieure de l'Electronique et de ses Applications, 95014 Cergy Pontoise, France.

H. Leier is with the Daimler Benz Research Center, D-7900 Ulm, Germany Publisher Item Identifier S 0018-9480(02)00019-4. 